\title{
Erratum zu: Freiwilliger Verzicht auf Nahrung und Flüssigkeit um das Sterben zu beschleunigen
}

\section{Eine Stellungnahme der österreichischen Palliativgesellschaft (OPG)}

\author{
Angelika Feichtner - Dietmar Weixler · Alois Birklbauer
}

Online publiziert: 24. April 2018

(c) Springer-Verlag GmbH Austria, ein Teil von Springer Nature 2018

\section{Erratum zu:}

Wien Med Wochenschr 2018

https://doi.org/10.1007/s10354-018-0629-z

Beim Hinweis zur Information der deutschen Bundesärztekammer [31] ist uns leider ein Fehler unterlaufen, den wir hiermit korrigieren möchten.

Im Artikel steht:

Entspricht es dem Patientenwillen, darf das Ster-

ben auch durch den Verzicht auf Nahrung und

Flüssigkeit ermöglicht werden.

Der Originaltext zu den Grundsätzen der Bundesärztekammer zur ärztlichen Sterbebegleitung lautet jedoch:

Darüber hinaus darf das Sterben durch Unterlassen, Begrenzen oder Beenden einer begonnenen medizinischen Behandlung ermöglicht werden, wenn dies dem Willen des Patienten entspricht. Dies gilt auch für die künstliche Nahrungsund Flüssigkeitszufuhr. (Bundesärztekammer. Grundsätze der Bundesärztekammer zur ärztlichen Sterbebegleitung. Dtsch Arztebl 2011; 108: A346-A348)

Wir bedauern diesen Fehler.

Angelika Feichtner, Dietmar Weixler, Alois Birklbauer

\section{Literatur}

31. Bundesärztekammer. Grundsätze der Bundesärztekammer zur ärztlichen Sterbebegleitung. Dtsch Arztebl. 2011;108(7):346-8.

Die Online-Version des Originalartikels ist unter https://doi. org/10.1007/s10354-018-0629-z zu finden.

A. Feichtner (ه)

Arbeitsgruppe „Ethik in Palliative Care“, Österreichische Palliativgesellschaft (OPG), Universitätsklinik für Innere Medizin 1, Medizinische Universität Wien, Wien, Österreich angelika.feichtner@gmx.net

D. Weixler

Landesklinikum Horn, Horn, Österreich

A. Birklbauer

Johannes-Kepler-Universität Linz, Linz, Österreich 